\title{
An Efficient Automated Tool for Design Time Reusability
}

\author{
Neeraj \\ Department of Computer Science \& Engineering \\ M.M University \\ Mullana (Ambala) \\ Haryana, INDIA
}

\begin{abstract}
The development of new applications based on the existing design and code increases software quality. This work shows how design reuse increases software quality. It is important in the development of new software version from the existing applications. It enables the reuse of significant portion of the existing design. Software Reuse is one of the most beneficial aspects of Change Management. This concept provides different kind of modular reusability. The proposed work is about to develop an efficient automated tool for Design Time Reusability to generate different Software Entity Relationship Diagrams (E-R Diagram) and Database creation. Real world situations are captured through Entity Relationship diagrams (E-R Diagram). A key component of the design process in Software development life cycle (SDLC) is the conversion of Entity Relationship Diagrams (E-R Diagram) into Relational model. The mappings are incorporated during the conversion process and a database design is achieved. Here a methodology is described for the design of a Relational database based on the E-R model by performing the reusability of Requirement Phase Design. The designed tool will be used to automate the process of mapping E-R Diagram into relational model and to generate (Data Definition Language) DDL statement for each relation.
\end{abstract}

\section{Keywords}

E-R Diagram, SDLC, DDL

\section{INTRODUCTION}

Software reusability refers to design features of software that enhance its suitability for reuse, this can save time and further development costs. For example: Every time a new customer purchases a product from a company their data can be added to a database via a form. This form will be reused on a number of occasions to add more customer data to the system. This may happen every minute, hour, week or year. The form is therefore reused throughout the lifecycle of the database system.

The development of new applications based on the existing design and code increases software quality. This work shows how design reuse increases software quality. It is important in the development of new software version from the existing applications. It enables the reuse of significant portion of the existing design.

The state of the practice in software engineering has been, by far, to blindly satisfy the given requirements. Significant work has been done on requirements Verification, that is, to ascertain that a final design or product truly satisfies the given requirements. This implies that once a set of requirements are given, and accepted, they are never questioned. The goal of

\author{
Ashish Oberoi \\ Department of Computer Science \& Engineering \\ M.M University \\ Mullana (Ambala) \\ Haryana, INDIA
}

the software engineer is to satisfy them, completely and correctly.

\section{RELATED WORK}

William, Frakes et al. [1] performed a work, "Software Reuse Research Status and Future". This paper summarizes software reuse research, discusses major research contributions and unsolved problems, provides pointers to key publications and introduces four papers selected from the eight international conferences on software reuse.

Xiang Luo et al. [2] performed a work, "On Improving Change Management Process for Enterprise IT Services". In this paper, Author consider the Change Management Process for Enterprise IT services with the goal of improving the efficiency of this process, i.e., minimizing change completion time and maximizing the "change capacity".

EnzhaoHu et al. [3] performed a work, "IT Project Change Management". This paper analyses the reasons why the project changes, the impact of project change and the scope of the personnel involved, proposes a change management solution.

Meyer.B et.al [4] performed a work".NET is Coming", this paper discusses the emergence of Microsoft's.net platform for $\mathrm{xml}$ based web services.NET has a central role in Microsoft strategy to integrate the internet, web services, building block services, numerous tools for developers and many other features.

Alonso.O et.al [5] performed a work "Generating Text Search Applications for databases" In this paper to show Reusable domain based assets the author shows how to connect a domain analysis tool output to a program generator and then to a domain implementations input.

Milli.H et.al [6] performed a work "Reusing Software: Issues and Research Directions". In this paper the implications of Reuse on the production with an emphasis on the technical challenges is discussed. In this both the issues are discussed with a focus on application generator.

Gomaa.H et.al [7] performed a work "Methods and tools for the automated Configuration of Distributed Applications from reusable Software Architectures and Components". In this paper a reuse oriented perspective is taken to design and implement configurable distributed applications.

Leurox .D et.al [8] performed a work "Rational Software Architect: A Tool for Domain Specific Modeling". In this paper rational software architecture is defined. In this paper it is shown that RSA offers all the important features of the previous generation of Rational Modeling Tools while supporting a much wider range of model formats.RSA diagrams can be used in editing and displaying models 
derived from any EMF based metamodel. This paper describes how RSA and EMF provides these capabilities and explores some of the ways that IBM is currently exploiting them.

Fernando Barros et.al [9] performed a work, "Increasing software Quality through design reuse". This Paper gives a different dimension to software reuse based on topology. This kind of reuse is termed here by inheritance of topology and permits the use of existing software topologies to define new ones without incurring the cost of creating design from scratch.

Sarbjeet Singh et al. [10] "Software Engineering - Survey of Reusability Based on Software Component". Survey of reusability based on software components that provide the assistance to the developer in the development of software. Reusability of software is an important prerequisite for cost and time-optimized software development Work in software reuse focuses on reusing artifacts. The paper discusses the reusability concepts for Component based Systems and explores several existing metrics for both white-box and black box components to measure reusability directly or indirectly and presents the special requirements on software in this domain and Reusability is about building a library of frequently used components, thus allowing new programs to be assembled quickly from existing components. ComponentBased Systems (CBS) have now become more generalized approach for application development.

Collins .S.T et.al [11] performed a work "Analyzing Product Development Task Networks to Examine Organizational Change". This paper uses network analysis to study task interactions in the development process at a small engineering company. In this paper small company organizational changes are examined by comparing its Pdp network properties at two points in time.

Dounias Habouba et al. [12] performed a work, "Engineering Change Management and Decision-Making Assistance Using Software Agent". This paper proposes an agent-based approach that ensures the propagation of an engineering change towards the external systems concerned. It also checks if the engineering change proposed for a mechanical product does not create any inconsistency with the constraints from the various disciplines involved. Each discipline is represented by an expert agent.

\section{PROPOSED SYSTEM}

\subsection{Algorithm}

This algorithm is about to map the E-R Diagram into the Relational model and to generate the DDL statement for each relation. The following algorithm explains the proposed work: Step 1 Identify the entities and the attributes

Step 2 Enter the user input

Step 3 Select the entities and their relationships

Step 4 The E-R Diagram is created

Step 5 Perform the mapping

5.1 Map regular entities

5.2 Map weak entities

5.3 Map binary relationship
5.4 Map unary relationships

5.5 Map ternary relationships

5.6 Map super type/sub type relationships

Step 6 the relational model is generated

Step 7 for $\mathrm{i}=1$ to $\mathrm{n}$

Step 8 Select the data type and width of attributes

Step 9 Generate the DDL statement and show the result

Step 10 Save the DDL statements

\subsection{Proposed Flow-Chart}

The proposed work is about to develop an efficient automated tool for design time reusability. This tool will map the E-R Diagram into the Relational model in automated way. This flow chart defines the flow of instructions from top to bottom.

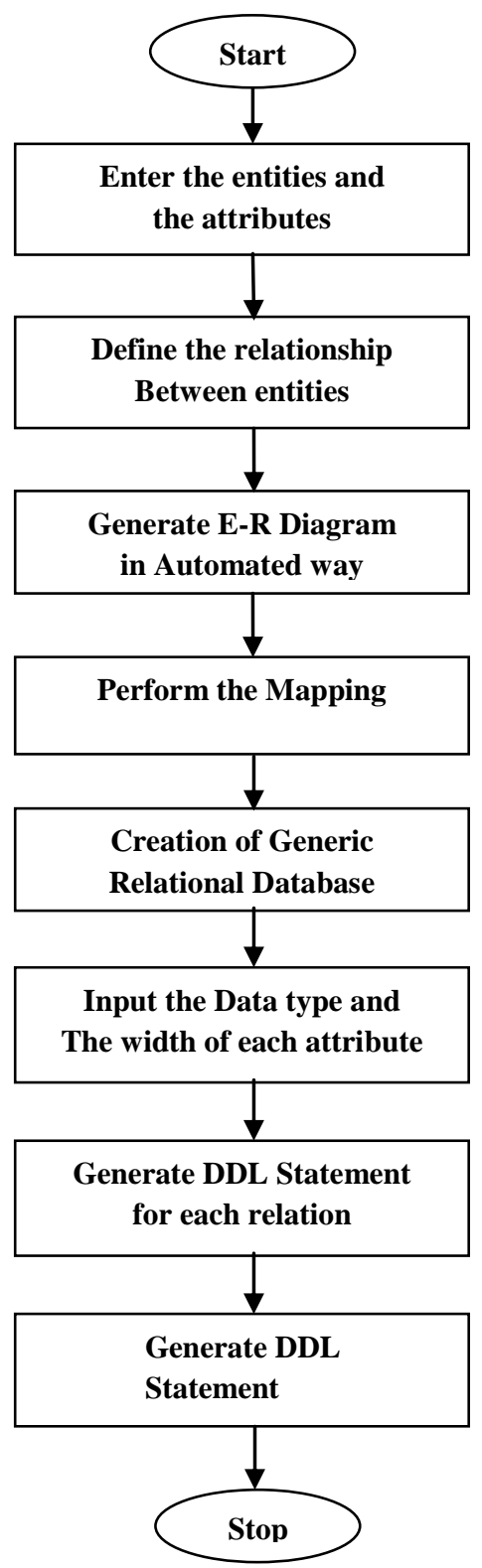

Fig 1: Proposed Flowchart

\section{RESULT AND DISCUSSION}

This work is implemented by using Visual studio .net. The proposed work is about to develop an efficient automated tool for Design Time Reusability. This tool can be used to 
generate different Software E-R Diagram and Database creation. Real world situations are captured through Entity Relationship diagrams. Here a methodology is described for the design of a Relational database based on the E-R model by performing the reusability of Requirement Phase Design. The mappings are incorporated during the conversion process and the database design is achieved. The designed tool will be used to automate the process of mapping E-R Diagram into Relational model and to generate DDL statement for each relation.

This work is basically divided into four main phases:

- User input

- Creation of E-R Diagram

- Mapping

- Generation of DDL statement

In this work first of all the basic E-R constructs like entities, their attributes, primary keys etc are find out from the given problem. During the first phase the user will enter these E-R constructs as an input to the form 1 for the generation of E-R Diagram

During the second phase of this work the user will define the relationship between the entities, and also define the type of relationship and the name of relationship. And as a result of this phase the E-R Diagram is obtained.

During the third phase the mapping of E-R Diagram into the Relational model is done and as a result the generic Relational model is obtained.

During the fourth phase the DDL statement for each relation is generated. And these DDL statements can also be saved.

\subsection{Comparison with the conventional methods:}

The manual transformation of E-R Diagram into Relational model leads to several problems.

Conventional way of transformation is more complex specifically if there is large number of entities and attributes and those results in large number of relationships among entities.

Transformation when carried out manually can be time consuming, prone to errors and costly, since it needs high skilled personnel. Errors creep into table structures during the transformation of E-R Diagrams to Relational schema.

Thus, automating the process of transformation is the only solution to eliminate the drawbacks of manual transformation.

\subsection{Real life Problem to Show the Difference (Library Management System)}

Library management refers to the issues involved in managing a library. In the library Management system, the following entities and attributes can be identified.

Book - the set all the books in the library. Each book has a Book-id, Name, Author, Issue date, Return date as its attributes.

Member - the set all the library members. The member entity is described by the attributes Member_id, Name, Address, Phone no, Publisher - the set of all the publishers of the books. Attributes of this entity are Pub_id, Name, Address,
Assumptions: A publisher publishes a book. Members borrow the book.

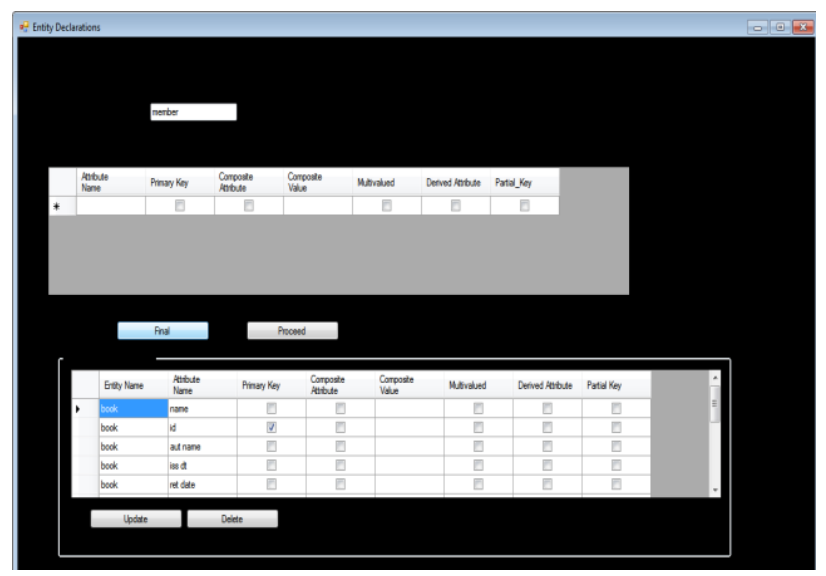

Fig 2: User Input

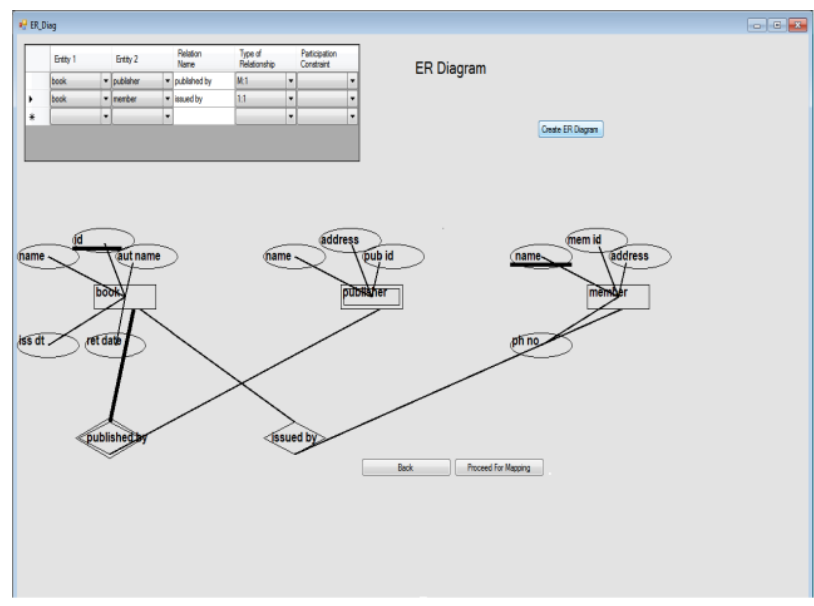

Fig 3: E-R Diagrams

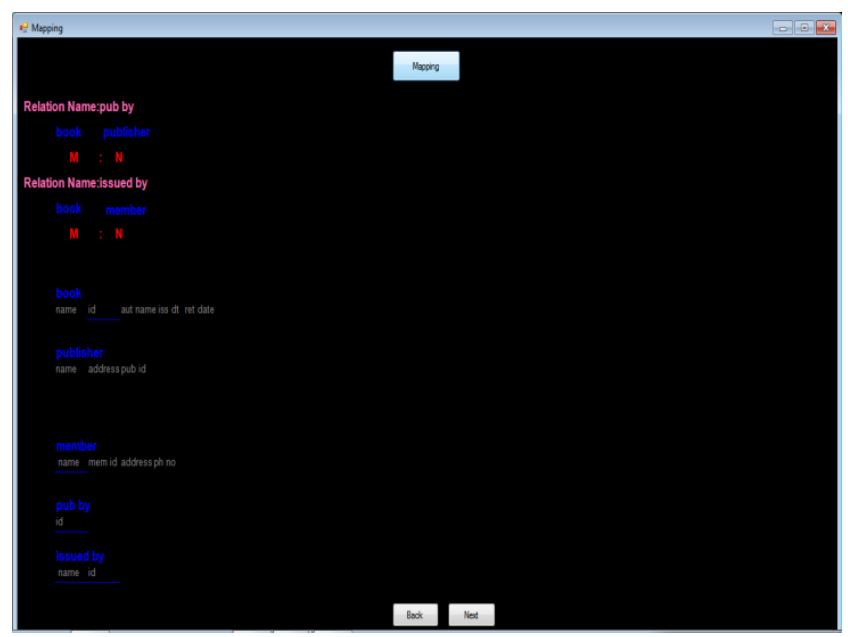

Fig 4: Mapping of E-R Diagram into Relational model 


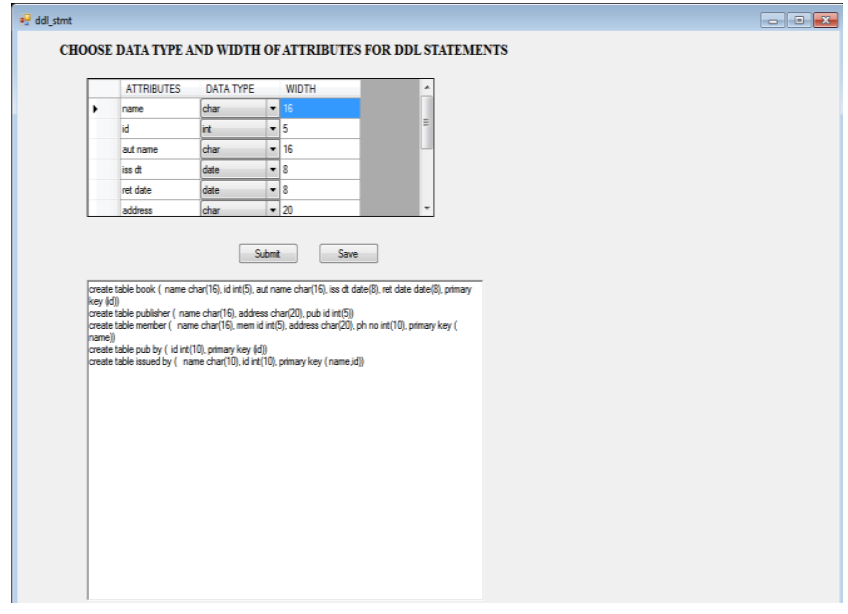

Fig 5: Generation of DDL Statements

\section{CONCLUSION \& FUTURE WORK}

This work deals with the one important aspect of change management called design reusability. The work includes the Automation of Generation of E-R Diagram. The work can be implemented on any real life database design project. The work covers the requirement phase and it will perform the database design of the real life software projects. This automated tool is efficient enough to map the E-R Diagram to effective Relation Database and will generate DDL statements for each relation.

As future work the work can be evaluated with larger applications and also the benefits of design time reuse in software quality can be measured quantitatively.

\section{REFERENCES}

[1] Milli.H, Milli.H, "Reusing Software: Issues and Research", in the proceedings of International Conference on reuse, vol21 (6), pp.528-562, 1995

[2] Gomaa.H , farrukh G.A, "Methods and Tools for the Automated Configuration of Distributed Applications from Reusable Software Architectures and Components", in the proceedings of international Conference on Software reuse, vol146 (6), pp.277-290, 1999
[3] Meyer .B, ".NET is coming", in the conference proceedings of International on information and Software Technology, vol34 (8), pp.92-97, 2001

[4] Alonso.O, "Generating Text Search Applications for Databases". In the proceedings of International Symposium on empirical Software engineering, vol20 (3), pp.98-105, 2003

[5] William. B, Frakes, "Software Reuse Research: Status and Future", in the proceedings of International Conference on Software Reuse, vol 31(7), pp.529-536, 2005

[6] Leurox. D, Nelly, "Rational Software Architect : A Tool for domain specific Modeling", in the proceedings of IBM system Journal,vol45(3), pp.555-568, 2006

[7] Xing Luo. "On Improving Change Management Process for Enterprise IT Services", in the Proceedings of International Conference on Services Computing, vol2 (7), pp. 341-345, 2008

[8] Enzhao Hu, “IT Project Change Management", in the Proceedings of International Symposium on Computer Science and Computational Technology,vol1(5), pp.417-420, 2008

[9] Sarbjeet Singh Manjit thapa, "Survey of Reusability Based on the software component", in the proceedings of international Journal of Computer Applications,vol8(12),pp.0975-8887,2010

[10] Fernando Barros, "Increasing Software Quality Through Design Reuse", in the proceedings of International Conference on reuse, pp.236-241, 2010

[11] Bradley, Collins. S.T, “Analyzing Product Development Task Networks to Examine Organizational Change", in the proceedings of International journal of computer science and network security, vol57(3), pp.513-525, 2010

[12] Suma V, Gopalakrishnan T.R, “ impact Analysis of Inspection process for effective Defect Management in software development", in the proceedings of American Society for quality Journal,vol12(2), pp.311-315, 2010

[13] Habouba. D, "Decision Making Assistance in Engineering-Change Management Process", in the proceedings of International Conference on System Sciences,vol41(3), pp.344-349, 2011 